\title{
Nomogram Containing Simple Routine Clinical and Biochemical Parameters Can Predict Pathological Ventricular Remodeling in STEMI Patients
}

\section{Ozren Vinter}

Klinicki bolnicki centar Sestre milosrdnice https://orcid.org/0000-0002-4236-7594

Kresimir Kordic ( $\square$ kordic.kresimir@yahoo.com )

Klinicki bolnicki centar Sestre milosrdnice https://orcid.org/0000-0002-9707-6946

Iva Klobucar

Klinicki bolnicki centar Sestre milosrdnice

Ivo Darko Gabric

Klinicki bolnicki centar Sestre milosrdnice

Marko Boban

Klinicki bolnicki centar Sestre milosrdnice

Matias Trbusic

Klinicki bolnicki centar Sestre milosrdnice

\section{Research article}

Keywords: acute myocardial infarction, left ventricular remodeling, end-diastolic volume, end-systolic volume

Posted Date: August 28th, 2020

DOI: https://doi.org/10.21203/rs.3.rs-60127/v1

License: (ㅇ) (1) This work is licensed under a Creative Commons Attribution 4.0 International License. Read Full License 


\section{Abstract}

BACKGROUND: Heart failure is the leading cause of worldwide morbidity and mortality, with ischemic heart disease being one of the most important etiologic factors. Heart failure develops due to ventricular remodeling, which leads to increases in left ventricular end-systolic and end-diastolic volumes.

METHODS: We conducted a prospective observational study to find which parameters could predict the risk of pathological left ventricular remodeling in the early stage of myocardial infarction.

RESULTS: We created a nomogram based on routinely used blood tests and vital parameters which had the highest correlation with pathological ventricular remodeling. The nomogram included NTproBNP value 12 hours after reperfusion, AST value 12 hours after reperfusion, systolic blood pressure value on admission and culprit coronary artery. We performed ROC analysis which found great predictive value of the nomogram. Area under curve (AUC) was $0,907(95 \% \mathrm{Cl} 0,842-0,973)$. Nomogram value of -3.54 had sensitivity of $91.4 \%$ and specificity of $74.0 \%$.

CONCLUSION: We believe that this nomogram, once validated, could offer a widely available, low-cost option that would help identify patients at risk of developing pathological left ventricular remodeling and achieve this at a very early stage of myocardial infarction ( 12 hours after reperfusion is achieved).

\section{Background}

Heart failure is the leading cause of worldwide morbidity and mortality. Atherosclerotic coronary artery disease is the most important promoting factor of myocardial infarction. Due to advances in treatment, number of patients who survive myocardial infarction is continually increasing, some of whom eventually develop heart failure.

Development of heart failure occurs due to ventricular remodeling, which includes structural, functional, cellular, and molecular changes involving cardiac myocytes and the interstitial collagen matrix. Processes described are initially compensatory and driven to preserve cardiac output. Pathologic remodeling may occur with pressure overload or volume overload, or following cardiac injury (eg. myocardial infarction). Following myocardial infarction, remodeling leads to ventricular dilation and increased oxygen demand $(1,2)$. As dilatation progresses, it leads to further haemodynamic consequences. Consequent alterations in geometry lead to mitral regurgitation (3), which also contributes to the development of ischemic cardiomyopathy.

Multiple therapeutic targets exist to stop adverse remodeling and promote reverse remodeling, including early revascularization, optimal medical therapy with neurohormonal antagonists, and cardiac resynchronization therapy in appropriately selected patients.

The aim of this study was to find which parameters could predict the risk of pathological left ventricular remodeling in the early stage of myocardial infarction.

\section{Materials And Methods}


We included 101 patient diagnosed with first episode of ST-elevation acute myocardial infarction. Acute myocardial infarction was defined according to European society of cardiology guidelines. Inclusion criteria were also percutaneous coronary intervention performed within 12 hours of onset of chest pain and age between 18-79 years. The study was approved and overseen by the hospitals Ethics committee board. All patients were informed of the study details by one of the authors and signed a written consent form which was approeved and reviewed by the Ethics comitee board. We excluded patients with known cardiomyopathy (regardless of etiology), chronic renal insufficiency stage IV and V, and intolerance of lipophilic ACEI or ARB. All included patients received standard therapy for myocardial infarction (ACEI/ARB, beta-blocker, statin, acetylsalicylic acid, clopidogrel/ticagrelor).

All blood tests and vital parameters were collected at baseline, 6 and 12 hours following PCI. NTproBNP was also collected 1 year following PCl.

Transthoracic echocardiography was performed within 5 days from myocardial infarction and 1 year later. Pathological ventricular remodeling was defined as EDVi (left ventricular end-diastolic volume index) > $79 \mathrm{ml} / \mathrm{m} 2$ for men, or EDVi $>71 \mathrm{ml} / \mathrm{m} 2$ for women and/or ESVi (left ventricular end-systolic volume index) > $32 \mathrm{ml} / \mathrm{m} 2$ for men, and ESVi $>28 \mathrm{ml} / \mathrm{m} 2$ for women, according to normal reference ranges for cardiac chambers size from NORRE study (4).

Coronary angiography was performed within 12 hours from onset of chest pain. All procedures were performed with transradial approach. Only culprit lesion was revascularized during primary $\mathrm{PCl}$, other significant lesions (evaluated by the operator who performed primary $\mathrm{PCl}$ ) were revascularized within 3 months.

The covariates included were age, systolic blood pressure on admission, diastolic blood pressure on admission, Killip class on admission, heart rate on admission, culprit coronary artery, number of arteries with signficant stenosis (defined as $>70 \%$ ), SYNTAX score, pain to baloon time, diabetes, weight, BMI, dyslipidaemia, smoking status, previous medical therapy, medical therapy on dismissal, hypertension, prior cerebrovascular disease, $\mathrm{CK}$ value at admission, $6 \mathrm{~h}$ post $\mathrm{PCl}$ and $12 \mathrm{~h}$ post $\mathrm{PCl}$, hsTNI at admission, $6 \mathrm{~h}$ post $\mathrm{PCl}$ and $12 \mathrm{~h}$ post $\mathrm{PCl}, \mathrm{AST}$ value at admission, $6 \mathrm{~h}$ post $\mathrm{PCl}$ and $12 \mathrm{~h}$ post $\mathrm{PCl}$, ALT value value at admission, $6 \mathrm{~h}$ post $\mathrm{PCl}$ and $12 \mathrm{~h}$ post $\mathrm{PCl}, \mathrm{LDH}$ value value at admission, $6 \mathrm{~h}$ post $\mathrm{PCl}$ and $12 \mathrm{~h}$ post $\mathrm{PCl}$, glucose plasma value value at admission, $6 \mathrm{~h}$ post $\mathrm{PCl}$ and $12 \mathrm{~h}$ post $\mathrm{PCl}, \mathrm{HbA} 1 \mathrm{C}$ during hospitalisation, NTproBNP value value at admission, $6 \mathrm{~h}$ post $\mathrm{PCl}$ and $12 \mathrm{~h}$ post $\mathrm{PCl}$, and 1 year after myocardial infarction.

Descriptive statistics was initially performed. Data distribution was analyzed by the Kolmogorov-Smirnov test. Most of the variables did not follow the normal distribution. Continuous variables were expressed as mean and standard deviation, but were analyzed by nonparametric tests. Independent samples were compared by the Kruskal-Wallis or Mann-Whitney test. Dependent continuous variables were analyzed by the Wilcoxon test. Categorical variables were compared by $\chi$-square test with Yates correction. The analysis of the correlation between individual parameters was done by Spearman correlation and binary logistic regression. Binary logistic regression was also used to analyze the interrelationship of the variables and to correct the correlation for the disruptive factors. When performing multivariate analysis, a model of backward stepwise conditional regression was used. The aim of this model was to find out which independent variables are independently related to the dependent variable, and the same was used in all forms of regression. ESVi was used as a dependent variable in the multivariate analysis, and a maximum of 6 variables were used in each model, which 
is appropriate to the sample size. Only variables that showed a statistically significant difference in univariate analysis were included in the multivariate analysis. Since variables that do not follow the normal distribution were used in the multivariate analysis, all analyzes on logarithmically transformed data were repeated. Since the same results were obtained, only the results obtained by the analysis of "raw" data are shown. In order to relate the predictive value of each parameter that proved to be an independent predictive factor, a regression equation composed of non-standardized coefficients of multivariate analysis for each individual predictive factor was constructed. Finally, reciever operating characteristic (ROC) analysis was used to analyze the predictive value of an individual parameter, or regression equation in the prediction of elevated ESVi.

Statistical analysis was performed with SPSS software version 20.0. All of the tests were considered statistically significant if $p$-value $<0.05$.

\section{Results}

We included 101 patient diagnosed with first episode of ST-elevation acute myocardial infarction. Primary percutaneous coronary intervention was performed within 12 hours of onset of chest pain.

Age, sex, Killip class, systolic blood pressure, heart rate and heart rhythm were recorded at admission. AST, ALT, CK, LDH, hs-troponin I and NTproBNP were collected at baseline, 6 and 12 hours following PCI. NTproBNP was also collected 1 year following PCI. Only variables that showed a statistically significant difference in univariate analysis were included in the multivariate analysis. (Tables 1, 2, 3 and 4).

In order to unify the predictive value of each parameter, a regression equation was made using nonstandardized coefficients for each parameter:

(NTproBNP_12 × 0,017 + AST_12 ×0,008 - SBP × 0,035) - 2,005 (for RCA) or - 4,949 (for ACx)

We performed ROC analysis which found great predictive value of the nomogram. Area under curve (AUC) was 0,907 (95\% Cl 0,842-0,973). Nomogram value of -3.54 had sensitivity of $91.4 \%$ and specificity of $74.0 \%$.

(Fig. 1.)

\section{Discussion}

The nomogram we created is based on routinely used blood tests and vital parameters which had the highest correlation with pathological ventricular remodeling. It included NTproBNP value 12 hours after reperfusion, AST value 12 hours after reperfusion, systolic blood pressure value on admission and culprit coronary artery. $\mathrm{ROC}$ analysis found great predictive value of the nomogram.

NTproBNP value is considered to be the most valuable and reliable biomarker for diagnosing HF and cardiac dysfunction. It is released to the bloodstream by myocytes undergoing wall tension due to volumetric or pressure overload. (5) It is well known that acute ischemic heart disease is associated with an elevation of BNP levels, which might reflect the severity of LV dysfunction (6). NTproBNP together with systolic blood pressure on admission could represent the Killip classification system, which is using physical examination and the development of heart failure in order to predict and stratify the risk of mortality after an acute myocardial infarction. AST and ALT elevations are common in myocardial infarction. Both markers are correlated with CK- 
MB area under the curve, but independently associated with worse mortality and clinical outcomes (7). Perhaps the biggest surprise in our nomogram is better correlation of pathological ventricular remodeling present 1 year after the myocardial infarction with AST than with CK (when CK is included instead of AST predictive value drops slighty, to approximately $88 \%$ ). This can be attributed to statistical "glitch" due to low patient sample, but we believe that, since AST proved to be elevated after ischemic cell death of several other tissues, including kidney, skeletal muscle and brain, better correlation of AST is due to it being a marker of ishaemic end organ damage in acute coronary syndrom and not dominantly a marker of myocardial lesion (8-12). It is also noted that myocardial infarction affecting left anterior descending coronary artery is associated with the highest risk of adverse clinical outcomes because of the large amount of myocardial territory supplied by the LAD compared to other coronary arteries (13).

All the parameters of the nomogram are routinely used and they add no extra cost. Their correlation has a great diagnostic accuracy in predicting left ventricular remodeling.

In our study, we used the absolute value of ESVi and EDVi to define pathological ventricular remodeling. Prospective study of Farah et al. included 66 patients with anterior infarction, with aim to analyze left ventricular remodeling at 6 months follow-up. Ventricular remodeling was defined as an increase of $10 \%$ in endsystolic or end-diastolic diameter (14). $58 \%$ of patients presented with ventricular remodeling, which is more than in our study (39.6\%). It can be explained by the fact that the above study included only patients with anterior myocardial infarction, who are at highest risk of developing pathological ventricular remodeling. When analyzing the subgroup of patients with anterior infarction from our study, the results are similar (61.7\% patients with anterior infarction developed pathological ventricular remodeling), despite the fact that the same criteria were not used to define pathological ventricular remodeling. In the study of Zaliaduonyte-Peksiene et al. remodeling was defined as 20\% increase in EDV within 6 months from myocardial infarction. (15) The prevalence of pathological ventricular remodeling was $34.7 \%$, which is similar to our results. Also, in study of Park et al., which included 50 patients with anterior-wall acute myocardial infarction to test whether longitudinal strain can be a useful predictor of left ventricular remodeling after reperfusion therapy in acute myocardial infarction, an increase of $15 \%$ in end-diastolic diameter was used to define pathological remodeling (16). However, the prevalence of left ventricular remodeling was similar to that observed in our study. Thus, it can be concluded that the use of an absolute value (EDVi $>79 \mathrm{ml} / \mathrm{m} 2$ for men, or EDVi $>71 \mathrm{ml} / \mathrm{m} 2$ for women and/or ESVi > $32 \mathrm{ml} / \mathrm{m} 2$ for men, and ESVi > $28 \mathrm{ml} / \mathrm{m} 2$ for women) is equally valuable and sensitive for assessing the occurrence of pathological ventricular remodeling as well as the use of dynamic value (increase in EDV at follow up). In our study, all patients with an increased ESVi also had an increased EDVi, suggesting that ESVi is a better parameter for predicting pathological ventricular remodeling compared to EDVi, which is supported in other studies. Saito et al. analyzed data from 266 STEMI patients who underwent successful percutaneous coronary intervention (17). Patients were divided into 4 groups according to LVEDP and LV EF value (LVEDP $\geq 21 \mathrm{mmHg}$ and LVEF $\geq 55 \%$ ). The study found a statistically significant difference in ESVi in the group with elevated LVEDP and decreased EF compared to group with normal LVEDP and decreased EF LV. Patients in the group with elevated LVEDP had an increased risk of MACE and re-hospitalization, emphasizing the value and importance of ESVi in the prognosis of patients with myocardial infarction.

Limitations of our study are several, biggest one being a relatively small patient sample. Also, since coronary disease severity assesment was performed by operator performing primary PCl, there is certanly a degree of 
intraobserver variance in assesment of coronary artery disease in out patients.

In a recent study, Rodriguez-Palomares JF et al. concluded that assessment of left ventricular remodeling at 6 months does not increase the prognostic value of the principal cardiac magnetic resonance (CMR) derived variables provided by the early CMR (18). Since access to CMR is limited, we believe that this nomogram, once validated, could offer a widely available, low-cost alternative to predict patients at risk of developing pathological left ventricular remodeling and do so in a very early stage of myocardial infarction (12 hours after reperfusion is achieved).

\section{Conclusion}

We created a nomogram based on routinely used blood tests and vital parameters with great diagnostic accuracy in predicting left ventricular remodeling. We believe that this nomogram, once validated, could offer a widely available, low-cost option to predict patients at risk of developing pathological left ventricular remodeling and do so in a very early stage of myocardial infarction (12 hours after reperfusion is achieved).

\section{List Of Abbreviations}

B - non-standardized coefficient

SE - standard error

OR - odds ratio

$\mathrm{Cl}$ - confidence interval

SBP - systolic blood pressure

HR - heart rate

EDVi - left ventricular end-diastolic volume index

ESVi - left ventricular end-systolic volume index

\section{Declarations}

\section{Ethics approval and consent to participate}

The study was overseeen and approeved by the hospital's Ethics committee bord (UHC Sisters of Mercy). Also, all patients signed a written consent form which incliuded study details. Prior to signing consent form and study details were explained to the patient by one of the investigators.

\section{Consent for publication}

All patients signed a consent form for study participation, but none of the dana published contain any individual patient information or images that could reveal patients identity. 
Availability of data and materials

The datasets during and/or analysed during the current study are available from the corresponding author on reasonable request

\section{Competing interests}

The authors declare that they have no competing interests

\section{Funding}

Study was partially funded by the Croatian Ministry for science and education with a grant of 20,000 kn (app. $2,700 €$ ) which was used to purchase NTproBNP ELISA kits ( remainder was purchased by private, author funds). Ministry had no influence in study design and data proccessing.

\section{Authors' contributions}

OV and KK contributed to the initiation, planning and conduction of the study. IK and IDG helped to perform the analysis with constructive discussions. MB and MT substantively revised the study and all authors commented on previous versions of the manuscript. All authors read and approved the final manuscript

\section{Acknowledgements}

\section{Not applicable}

\section{References}

1. McKay RG, Pfeffer MA, Pasternak RC, Markis JE, Come PC, Nakao S, et al. Left ventricular remodeling after myocardial infarction: a corollary to infarct expansion. Circulation. 1986 Oct;74(4):693-702.

2. Bhatt AS, Ambrosy AP, Velazquez EJ. Adverse Remodeling and Reverse Remodeling After Myocardial Infarction. Curr Cardiol Rep. 2017;19(8):71.

3. Benjamin MM, Smith RL, Grayburn PA. Ischemic and functional mitral regurgitation in heart failure: natural history and treatment. Curr Cardiol Rep. 2014 Aug;16(8):517.

4. Kou S, Caballero L, Dulgheru R, et al. Echocardiographic reference ranges for normal cardiac chamber size: results from the NORRE study. Eur Heart J Cardiovasc Imaging. 2014;15(6):680-690.

5. Souza TMB, Cerqueira AMS Jr, Suerdieck JG, et al. Prognostic Value of NT-proBNP versus Killip Classification in Patients with Acute Coronary Syndromes. Arq Bras Cardiol. 2020;114(4):666-672.

6. Cao Z, Jia Y, Zhu B. BNP and NT-proBNP as Diagnostic Biomarkers for Cardiac Dysfunction in Both Clinical and Forensic Medicine. Int J Mol Sci. 2019;20(8):1820.

7. Lofthus DM, Stevens SR, Armstrong PW, Granger CB, Mahaffey KW. Pattern of liver enzyme elevations in acute ST-elevation myocardial infarction. Coron Artery Dis. 2012;23(1):22-30.

8. Steininger M, Winter MP, Reiberger T, et al. De-Ritis Ratio Improves Long-Term Risk Prediction after Acute Myocardial Infarction. J Clin Med. 2018;7(12):474. 
9. Gao M, Cheng Y, Zheng Y, Zhang W, Wang L, Qin L. Association of serum transaminases with short- and long-term outcomes in patients with st-elevation myocardial infarction undergoing primary percutaneous coronary intervention. BMC Cardiovasc. Disord. 2017, 17, 43.

10. Batin P, Wickens M, McEntegart D, Fullwood L, Cowley AJ. The importance of abnormalities of liver function tests in predicting mortality in chronic heart failure. Eur. Heart J. 1995, 16, 1613-1618.

11. Poelzl G, Ess M, Mussner-Seeber C, Pachinger O, Frick M, Ulmer H. Liver dysfunction in chronic heart failure: Prevalence, characteristics and prognostic significance. Eur. J. Clin. Invest. 2012, 42, 153-163.

12. Alvarez AM, Mukherjee D. Liver abnormalities in cardiac diseases and heart failure. Int. J. Angiol. 2011, 20, $135-142$.

13. Aboelkasem Ali Mousa M, Abdelsabour Abdallah M, Shamseddin Mohammad H, Ahmad Aly Youssef A. Early predictors of left ventricular remodeling after primary percutaneous coronary intervention. Egypt Heart J. 2018;70(4):403-407.

14. Farah E, Cogni AL, Minicucci MF, Azevedo PS, Okoshi K, Matsubara BB, et al. Prevalence and predictors of ventricular remodeling after anterior myocardial infarction in the era of modern medical therapy. Med Sci Monit Int Med J Exp Clin Res. 2012 May 1;18(5):CR276-81.

15. Zaliaduonyte-Peksiene D, Simonyte S, Lesauskaite V, Vaskelyte J, Gustiene O, Mizariene V, et al. Left ventricular remodeling after acute myocardial infarction: Impact of clinical, echocardiographic parameters and polymorphism of angiotensinogen gene. J Renin Angiotensin Aldosterone Syst. 2014 Sep;15(3):28693.

16. Park YH, Kang S-J, Song J-K, Lee EY, Song J-M, Kang D-H, et al. Prognostic value of longitudinal strain after primary reperfusion therapy in patients with anterior-wall acute myocardial infarction. J Am Soc Echocardiogr Off Publ Am Soc Echocardiogr. 2008 Mar;21(3):262-7.

17. Saito D, Nakanishi R, Watanabe I, Yabe T, Okubo R, Amano H, et al. Combined assessment of left ventricular end-diastolic pressure and ejection fraction by left ventriculography predicts long-term outcomes of patients with ST-segment elevation myocardial infarction. Heart Vessels. 2018 May;33(5):453-61.

18. Rodriguez-Palomares JF, Gavara J, Ferreira-González I, et al. Prognostic Value of Initial Left Ventricular Remodeling in Patients With Reperfused STEMI. JACC Cardiovasc Imaging. 2019;12(12):2445-2456.

\section{Tables}

Table 1. Multivariate analysis of clinical, anthropometric and anatomical parameters that showed a statistically significant difference in univariate analysis (except KILLIP because of unfavourable distribution). 


\begin{tabular}{|c|c|c|c|c|c|c|}
\hline & $\mathrm{B}$ & SE & $P$ & OR & \multicolumn{2}{|c|}{$95 \% \mathrm{Cl}$} \\
\hline \multicolumn{7}{|c|}{ Model 1 (sex, SBP, HR) } \\
\hline Male sex & 1,179 & 0,549 & 0,032 & 3,252 & 1,109 & 9,534 \\
\hline SBP & $-0,026$ & 0,012 & 0,027 & 0,974 & 0,952 & 0,997 \\
\hline $\mathrm{HR}$ & 0,043 & 0,017 & 0,010 & 1,044 & 1,010 & 1,078 \\
\hline \multicolumn{7}{|c|}{ Model 2 (Model 1 + SYNTAX) } \\
\hline Male sex & 0,923 & 0,568 & 0,105 & 2,516 & 0,826 & 7,668 \\
\hline SBP & $-0,022$ & 0,012 & 0,057 & 0,978 & 0,956 & 1,001 \\
\hline $\mathrm{HR}$ & 0,039 & 0,017 & 0,022 & 1,040 & 1,006 & 1,075 \\
\hline SYNTAX & 0,068 & 0,030 & 0,025 & 1,070 & 1,009 & 1,135 \\
\hline \multicolumn{7}{|c|}{ Model 3 (Model 1 + artery) } \\
\hline $\mathrm{SBP}(\mathrm{mmHg})$ & $-0,024$ & 0,012 & 0,039 & 0,976 & 0,954 & 0,999 \\
\hline \multicolumn{7}{|l|}{ Coronary artery } \\
\hline RCA vs. LAD & $-1,482$ & 0,513 & 0,004 & 0,227 & 0,083 & 0,621 \\
\hline$A C x$ vs. LAD & $-2,390$ & 0,820 & 0,004 & 0,092 & 0,018 & 0,457 \\
\hline \multicolumn{7}{|c|}{ Model 4 (Model $1+$ SYNTAX + artery) } \\
\hline $\mathrm{SBP}(\mathrm{mmHg})$ & $-0,021$ & 0,012 & 0,074 & 0,979 & 0,957 & 1,002 \\
\hline Coronary artery & & & 0,011 & & & \\
\hline RCA vs. LAD & $-1,256$ & 0,531 & 0,018 & 0,285 & 0,101 & 0,806 \\
\hline$A C x$ vs. LAD & $-2,006$ & 0,845 & 0,018 & 0,135 & 0,026 & 0,705 \\
\hline SYNTAX & 0,052 & 0,030 & 0,081 & 1,054 & 0,994 & 1,117 \\
\hline
\end{tabular}




\begin{tabular}{|lllllll|}
\hline \multicolumn{2}{|l|}{ Model 5 (SYNTAX + artery) } \\
\multicolumn{7}{|l|}{ Coronary artery } \\
\hline RCA vs. LAD & $-1,248$ & 0,515 & 0,015 & 0,287 & 0,105 & 0,789 \\
\hline ACX vs. LAD & $-2,051$ & 0,832 & 0,014 & 0,129 & 0,025 & 0,657 \\
\hline SYNTAX & 0,063 & 0,029 & 0,032 & 1,065 & 1,005 & 1,128 \\
\hline
\end{tabular}

B - non-standardized coefficient; SE - standard error; OR - odds ratio; $\mathrm{Cl}$ - confidence interval; SBP - systolic blood pressure; HR - heart rate

Table 2. Spearman correlation of clinical parameters included in multivariate analysis. *. The correlation is significant at the 0.05 level.

\begin{tabular}{|llllll|}
\hline \multirow{3}{*}{ Sex } & & Sex & SBP & HR & SYNTAX \\
& Coefficient & 1,000 & $-0,184$ & $-0,032$ & $0,205^{*}$ \\
\cline { 2 - 6 } & P & $\cdot$ & 0,066 & 0,753 & 0,042 \\
\hline \multirow{2}{*}{ HRP } & Coefficient &,- 184 & 1,000 & 0,085 & $-0,215^{*}$ \\
\cline { 2 - 6 } & P & 0,066 & $\cdot$ & 0,402 & 0,033 \\
\cline { 2 - 6 } & Coefficient & $-0,032$ & 0,085 & 1,000 & 0,114 \\
\cline { 2 - 6 } & P & 0,753 & 0,402 & $\cdot$ & 0,268 \\
& Coefficient & $0,205^{*}$ & $-0,215^{*}$ & 0,114 & 1,000 \\
\cline { 2 - 6 } & P & 0,042 & 0,033 & 0,268 &. \\
\hline
\end{tabular}

Table 3. Multivariate analysis of laboratory parameters that had the strongest predictive value in univariate analysis and their combination with previous clinical, anthropometric and anatomical parameters. 


\begin{tabular}{|c|c|c|c|c|c|c|}
\hline & B & SE & $\mathrm{P}$ & OR & \multicolumn{2}{|l|}{$95 \% \mathrm{Cl}$} \\
\hline \multicolumn{7}{|c|}{ Model 1 (BNP, troponin, CK, AST, ALT, LDH 12 hours after reperfusion) } \\
\hline BNP_12 & 0,011 & 0,005 & 0,023 & 1,011 & 1,001 & 1,020 \\
\hline AST_12 & 0,009 & 0,002 & $<0,001$ & 1,009 & 1,004 & 1,013 \\
\hline \multicolumn{7}{|c|}{ Model 2 (BNP_12, AST_12 + sex, SBP, HR ) } \\
\hline BNP_12 & 0,012 & 0,005 & 0,020 & 1,012 & 1,002 & 1,021 \\
\hline AST_12 & 0,007 & 0,002 & 0,002 & 1,007 & 1,003 & 1,012 \\
\hline SBP & $-0,033$ & 0,016 & 0,043 & 0,967 & 0,937 & 0,999 \\
\hline HR & 0,039 & 0,022 & 0,069 & 1,040 & 0,997 & 1,085 \\
\hline \multicolumn{7}{|c|}{ Model 3 (Model 2 + SYNTAX) } \\
\hline BNP_12 & 0,012 & 0,005 & 0,021 & 1,012 & 1,002 & 1,023 \\
\hline AST_12 & 0,007 & 0,002 & 0,005 & 1,007 & 1,002 & 1,011 \\
\hline SBP & $-0,034$ & 0,016 & 0,039 & 0,966 & 0,936 & 0,998 \\
\hline HR & 0,037 & 0,021 & 0,086 & 1,038 & 0,995 & 1,082 \\
\hline \multicolumn{7}{|c|}{ Model 4 (Model $1+$ SYNTAX + artery) } \\
\hline BNP_12 & 0,017 & 0,006 & 0,002 & 1,018 & 1,006 & 1,029 \\
\hline AST_12 & 0,008 & 0,003 & 0,007 & 1,008 & 1,002 & 1,014 \\
\hline SBP & $-0,035$ & 0,018 & 0,047 & 0,965 & 0,932 & 1,000 \\
\hline \multicolumn{7}{|c|}{ Coronary artery } \\
\hline RCA vs. LAD & $-2,005$ & 0,705 & 0,004 & 0,135 & 0,034 & 0,536 \\
\hline ACx vs. LAD & $-4,949$ & 1,876 & 0,008 & 0,007 & $<0,001$ & 0,280 \\
\hline
\end{tabular}


B - non-standardized coefficient; SE - standard error; OR - odds ratio; Cl - confidence interval; SBP - systolic blood pressure; HR - heart rate

Table 4. Correlation of individual laboratory parameters, Spearman correlation.

\begin{tabular}{|c|c|c|c|c|c|c|c|c|}
\hline & & BNP_12 & Trop_12 & CK_12 & ALT_12 & AST_12 & GUP_12 & LDH_12 \\
\hline \multirow[t]{2}{*}{ BNP_12 } & Coefficient & 1,000 & 0,006 & 0,005 & 0,016 & 0,060 & 0,043 & 0,036 \\
\hline & $\mathrm{P}$ & . & 0,955 & 0,962 & 0,882 & 0,576 & 0,722 & 0,741 \\
\hline \multirow{2}{*}{$\begin{array}{l}\text { Troponin } \\
\text { I_12 }\end{array}$} & Coefficient & 0,006 & 1,000 & $0,840^{\star *}$ & $0,739^{* *}$ & $0,820^{\star \star}$ & 0,176 & $0,819^{\star \star}$ \\
\hline & $P$ & 0,955 & . & $<0,001$ & $<0,001$ & $<0,001$ & 128 & $<0,001$ \\
\hline \multirow[t]{2}{*}{ CK_12 } & Coefficient & 0,005 & $0,840^{\star *}$ & 1,000 & $0,819^{\star *}$ & $0,947^{* *}$ & $0,240^{*}$ & $0,915^{\star \star}$ \\
\hline & $\mathrm{P}$ & 0,962 & $<0,001$ & . & $<0,001$ & $<0,001$ & 0,035 & $<0,001$ \\
\hline \multirow[t]{2}{*}{ ALT_12 } & Coefficient & 0,016 & $0,739^{\star *}$ & $0,819^{\star *}$ & 1,000 &, $877^{\star \star}$ & $0,229^{*}$ & $0,846^{* *}$ \\
\hline & $\mathrm{P}$ & 0,882 & $<0,001$ & $<0,001$ & . & $<0,001$ & 0,045 & $<0,001$ \\
\hline \multirow[t]{2}{*}{ AST_12 } & Coefficient & 0,060 & $0,820^{\star *}$ & $0,947^{* *}$ & $0,877^{\star *}$ & 1,000 & $0,252^{*}$ & $0,958^{\star \star}$ \\
\hline & $\mathrm{P}$ & 0,576 & $<0,001$ & $<0,001$ & $<0,001$ & & 0,027 & $<0,001$ \\
\hline \multirow[t]{2}{*}{ Glucose_12 } & Coefficient & 0,043 & 0,176 & $0,240^{*}$ & $0,229^{*}$ & $0,252^{*}$ & 1,000 & $0,320^{\star \star}$ \\
\hline & $P$ & 0,722 & 0,128 & 0,035 & 0,045 & 0,027 & . & 0,005 \\
\hline \multirow[t]{2}{*}{ LDH_12 } & Coefficient & 0,036 & $0,819^{\star *}$ & $0,915^{\star *}$ & $0,846^{* *}$ & $0,958^{\star \star}$ & $0,320^{\star *}$ & 1,000 \\
\hline & $\mathrm{P}$ & 0,741 & $<0,001$ & $<0,001$ & $<0,001$ & $<0,001$ & 0,005 & . \\
\hline \multicolumn{9}{|c|}{ * The correlation is statistically significant at the level 0,05} \\
\hline$* *$ The $c$ & & riani & tet & 0,01 & & & & \\
\hline
\end{tabular}

\section{Figures}




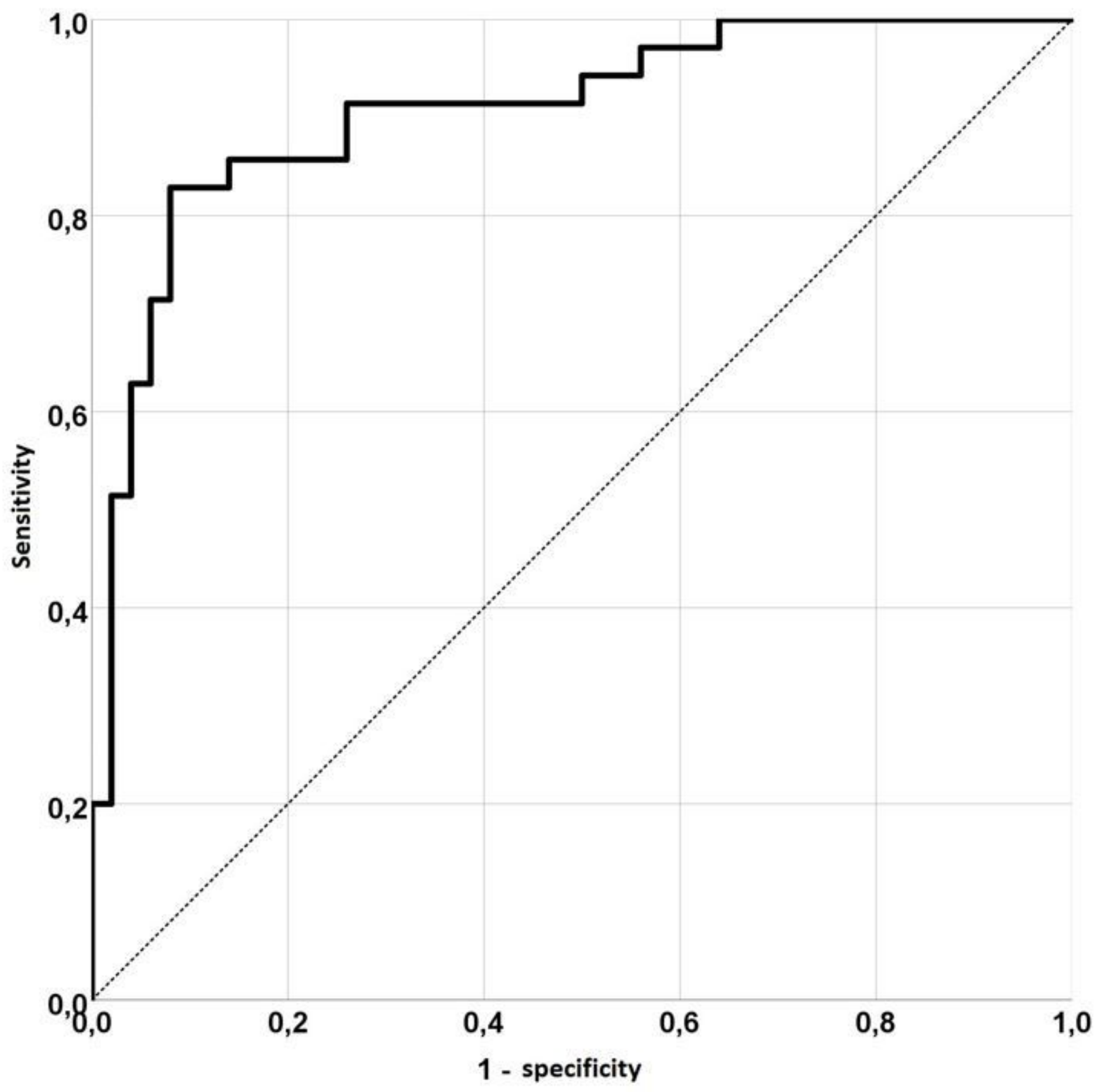

Figure 1

ROC curve showing diagnostic accuracy of the nomogram in predicting increased ESVi. 